\title{
Estamos Ensinando o que É Necessário sobre Doenças Reumatológicas?
}

\author{
Are We Teaching what We Need to about \\ Rheumatologic Diseases?
}

\author{
Pedro Albuquerque Rebello \\ Thiago Damasceno Corrêa ${ }^{I}$ \\ Igor Beltrão Duarte Fernandes ${ }^{I}$ \\ Cezar Augusto Muniz Caldas ${ }^{I, I I}$
}

PALAVRAS-CHAVE

- Educação Médica;

- Reumatologia;

- Escolas Médicas.

\section{KEYWORDS:}

- Medical Education;

- Rheumatology;

- Medical Schools.

Recebido em: 13/09/2014

Aprovado em: 10/03/2015

REVISTA BRASILEIRA DE EDUCAÇÃO MÉDICA

\section{RESUMO}

Condições musculoesqueléticas foram identificadas como a principal causa de dor crônica e incapacidade em todo o mundo. É necessário que o estudante de Medicina entre em contato com as doenças mais frequentemente observadas na prática diária, a fim de se minimizarem as dificuldades do futuro profissional no manejo destas condições. Objetivou-se determinar a prevalência das doenças reumáticas entre os pacientes atendidos no ambulatório de Reumatologia do Centro Universitário do Estado do Pará (Cesupa). Um estudo retrospectivo foi realizado por meio da análise de prontuários de pacientes atendidos de outubro de 2013 a abril de 2014. Os dados de 255 pacientes foram incluídos, sendo $223(87,5 \%)$ do sexo feminino, com idades de 47,69 $\pm 15,99$ anos. Dentre as 344 doenças diagnosticadas, houve predomínio de osteoartrite (23,54\%), seguida de artrite reumatoide (20,05\%), colagenoses $(17,44 \%)$ e reumatismo de partes moles $(15,69 \%)$. Conclui-se que o perfil de doenças com o qual os graduandos têm tido maior contato é, em parte, compatível com o perfil de afecções musculoesqueléticas mais comuns. Preocupa, porém, a baixa frequência de doenças como gota, cervicalgia e lombalgia, devendo a instituição otimizar oportunidades de contato com pacientes com tais doenças.

Musculoskeletal disorders have been identified as the main cause of chronic pain and incapacity all over the world. Medical students require contact with the most commonly observed diseases in daily clinical practice in order to minimize the difficulties they might face when handling these conditions as new professionals. The aim was to determine the prevalence of rheumatic diseases among patients seen at the Rheumatology Clinic of the Pará State University Center (CESUPA). A retrospective study was performed through analysis of the medical records of patients seen between October 2013 and April 2014. The data for 255 patients were included, 223 (87.5\%) of whom were female, and aged $47.69 \pm 15.99$ years. Of the 344 diseases diagnosed, the most prevalent was osteoarthritis (23.54\%), followed by rheumatoid arthritis (20.05\%), collagen disease (17.44\%) and soft-tissue rheumatism $(15.69 \%)$. It is concluded that the profile of diseases with which graduates have had most contact is partially compatible with the profile of the most common musculoskeletal disorders. However, there is concern regarding the low frequency of conditions such as gout, cervicalgia and low back pain, meaning the institution must optimize opportunities of contact with such patients. 


\section{INTRODUÇÃO}

Problemas relacionados ao sistema musculoesquelético, como a lombalgia aguda e a osteoartrite, representam o terceiro motivo mais frequente de busca por uma consulta com médicos generalistas. Estima-se que este tipo de enfermidade represente $15 \%$ do total de consultas na Atenção Primária ${ }^{1}$. Condições musculoesqueléticas foram identificadas como a principal causa de dor crônica e incapacidade ${ }^{2}$. Em razão destes fatores, acrescidos da expectativa de aumento da frequência das condições musculoesqueléticas devido ao envelhecimento da população, a formação do médico generalista, capaz de atuar na Atenção Primária com resolutividade sobre as afecções musculoesqueléticas mais comuns, deve ser objetivo das instituições de ensino. Os principais problemas na atenção ao sistema musculoesquelético têm sido estudados, e dentre eles destacam-se a acurácia do diagnóstico, solicitação de exames, medicações usadas e atrasos na referência para especialistas, muitos vezes de anos ${ }^{3,4}$.

Apesar da notável importância, pesquisas apontam que o ensino da Reumatologia está prejudicado. Em um estudo sobre graduação em Medicina, relatou-se que o tempo dedicado à disciplina é desproporcionalmente baixo quando relacionado com a frequência de queixas musculoesqueléticas encontradas na prática geral ${ }^{5}$. Além disso, mais de $80 \%$ dos avaliados se sentiram com baixo nível de confiança para realizar o exame musculoesquelético e $75 \%$ dos participantes desse mesmo estudo obtiveram aproveitamento igual ou inferior a $65 \%$ em uma prova de Reumatologia. Ainda segundo o mesmo estudo, o nível de competência dos graduandos em Medicina para resolver problemas musculoesqueléticos foi considerado inadequado ${ }^{5}$.

Há de se ressaltar que os serviços de saúde oferecidos em ambiente universitário tendem a ser de caráter especializado, muitas vezes incongruente com a prática diária do médico generalista. Doenças como artrite reumatoide, lúpus eritematoso sistêmico, esclerose sistêmica, síndrome de Sjögren, miosites inflamatórias autoimunes, vasculites e colagenoses infantis representam cerca de $66 \%$ dos diagnósticos de pacientes de hospital-escola, enquanto mais de $70 \%$ dos atendimentos em consultórios particulares estão relacionados com afecções de coluna, osteoartrites, doenças osteometabólicas, doenças microcristalinas, artropatias soronegativas, reumatismo de partes moles e casos não reumatológicos ${ }^{6}$. Hayata et al. ${ }^{6}$ destaca a necessidade de adequação do ensino da Reumatologia ao contexto especializado, enfatizando as doenças fora do espectro das colagenoses, sem, contudo, haver detrimento do ensino, pesquisa e avanços nas enfermidades autoimunes, cuja magnitude se tornou vitrine da especialidade.
Pelo exposto, objetivou-se determinar a prevalência das doenças reumatológicas entre os pacientes atendidos num serviço de referência em Reumatologia vinculado a um curso de graduação em Medicina, procurando verificar a adequação deste perfil às necessidades da população e dos futuros médicos.

\section{MÉTODO}

Trata-se de um estudo retrospectivo e descritivo, que representa a análise dos prontuários dos pacientes atendidos no período de outubro de 2013 a abril de 2014 no ambulatório de Reumatologia do Centro de Especialidades Médicas do Cesupa (Cemec). Os dados foram obtidos de acordo com um protocolo elaborado pelos próprios autores. O protocolo aplicado incluiu variáveis como: sexo, idade e diagnóstico(s) confirmado(s). Os casos de diagnósticos ainda não confirmados foram excluídos, assim como aqueles com informações incompletas ou ilegíveis. A coleta de dados ocorreu após o aceite institucional e a aprovação do Comitê de Ética em Pesquisa do Cesupa (parecer $n^{\circ} 189.320 / 2014$ ).

Os dados foram organizados em planilhas do Microsoft Excel 2010®. As variáveis categóricas foram apresentadas em valores absolutos e percentuais; as variáveis contínuas foram apresentadas através da média \pm desvio-padrão (DP). Para verificar as diferenças entre as proporções, foi utilizado o teste do Qui-Quadrado, considerando p-valor significativo $<0,05$.

\section{RESULTADOS}

Foram analisados 255 prontuários, sendo a maioria de pacientes do sexo feminino (87,5\%), com média de idade de 47,69 \pm 15,99 anos, com predomínio de pacientes na faixa etária dos 16 aos 60 anos (Tabela 1).

\begin{tabular}{|c|c|c|c|}
\hline \multicolumn{4}{|c|}{$\begin{array}{c}\text { TABELA } 1 \\
\text { Distribuição dos pacientes por sexo e faixa etária }\end{array}$} \\
\hline & $\mathbf{N}$ & $\%$ & $\mathrm{p}$ \\
\hline \multicolumn{4}{|l|}{ Sexo } \\
\hline Feminino & 223 & 87,5 & $<0,001^{*}$ \\
\hline Masculino & 32 & 12,5 & \\
\hline Total & 255 & 100 & \\
\hline \multicolumn{4}{|l|}{ Faixa etária } \\
\hline$<16$ anos & 5 & 2 & \\
\hline 16 a 60 anos & 194 & 76 & $<0,001^{*}$ \\
\hline$>60$ anos & 56 & 22 & \\
\hline Total & 255 & 100 & \\
\hline
\end{tabular}

Fonte: Protocolo de pesquisa. Nota: * Qui-Quadrado. 
Nos prontuários avaliados, foram diagnosticadas 344 doenças. A mais prevalente foi a osteoartrite ( $\mathrm{n}=81 ; 23,54 \%$ ), seguida de artrite reumatoide $(n=69 ; 20,05 \%)$, colagenoses $(n$ $=60 ; 17,44 \%)$ e reumatismo de partes moles $(n=54 ; 15,69 \%)$. Dentre os diagnósticos menos frequentes estão gota e cervicalgia, ambas com 6 casos $(1,74 \%)$, seguidas de lombalgia $(0,87 \%)$ e artrite idiopática juvenil (0,58\%) (Tabela 2).

\begin{tabular}{|lcc|}
\hline \multicolumn{4}{c}{ TABELA 2 } & & \\
\hline \multicolumn{2}{c}{ Frequência das doenças diagnosticadas } \\
\hline Osteoartrite & N & $\%$ \\
\hline Artrite reumatoide & 81 & 23,54 \\
\hline Colagenoses & 69 & 20,05 \\
\hline Reumatismo de partes moles & 60 & 17,44 \\
\hline Osteoporose & 54 & 15,69 \\
\hline Fibromialgia & 22 & 6,39 \\
\hline Espondiloartrites & 19 & 5,52 \\
\hline Outros & 14 & 4,06 \\
\hline Gota & 8 & 2,32 \\
\hline Cervicalgia & 6 & 1,74 \\
\hline Lombalgia & 6 & 1,74 \\
\hline Artrite idiopática juvenil & 3 & 0,87 \\
\hline Total & 2 & 0,58 \\
\hline
\end{tabular}

Fonte: Protocolo de pesquisa.

Dentre as colagenoses, o lúpus eritematoso sistêmico (LES) foi a mais frequente ( $n=46 ; 13,37 \%)$; no grupo das espondiloartrites, destaca-se a espondilite anquilosante, presente em $7(2,03 \%)$ pacientes.

\section{DISCUSSÃO}

Observa-se no presente estudo que doenças de importante relevância epidemiológica, como a osteoartrite e a artrite reumatoide (AR), têm feito parte do dia a dia do estudante de Medicina durante o estudo da Reumatologia. Porém, outras doenças, tão relevantes quanto essas, como artropatias por cristais, cervicalgia e lombalgia, contribuem pouco com o número de pacientes atendidos no ambulatório de Reumatologia do Cesupa.

Por se tratar de um serviço especializado, a tendência é que doenças como o LES e a AR contribuam com um número significativo de atendimentos no ambulatório de Reumatologia. Entretanto, por se tratar da formação de graduandos de Medicina, com ênfase no perfil do médico generalista, doenças como reumatismos de partes moles, osteoartrite, artropatias por cristais, cervicalgia e lombalgia precisam ser enfatizadas durante o curso.
O curso de Medicina do Cesupa é estruturado em metodologias ativas de aprendizado, com inserção precoce do aluno em campos de prática junto à comunidade, como ocorre desde o primeiro semestre do curso através dos Módulos de Interação em Saúde na Comunidade (Misc). No Misc, o aluno tem contato com pacientes com as doenças mais comuns, incluindo dores articulares, cervicalgia, lombalgia, osteoartrite e osteoporose. Desta forma, estes cenários podem contribuir significativamente para a aprendizagem sobre afecções do sistema musculoesquelético, sendo necessário que a instituição esteja atenta a garantir que professores/preceptores não especialistas em Reumatologia possam assegurar o nível de discussão adequado sobre a abordagem ao paciente com queixas do sistema locomotor e as principais afecções deste sistema ${ }^{7}$.

Segundo Hossie ${ }^{1}$, a educação adequada de graduandos de Medicina, dando-lhes noções gerais na área de Reumatologia, contribuiria de forma expressiva para diminuir a pressão sobre os cuidados secundários, além de permitir aos reumatologistas maior enfoque às doenças inflamatórias e àquelas que afetam o tecido conjuntivo. Neste contexto, é de substancial importância que o profissional desenvolva certas competências básicas, como coletar uma história adequada a respeito da queixa musculoesquelética do paciente, executar um exame físico relevante, além de ter conhecimento sobre Epidemiologia.

Mesmo se tratando de um serviço especializado, a ênfase na discussão das doenças mais comuns não pode ser negligenciada. Desta forma, a presença da osteoartrite como diagnóstico predominante no ambulatório de Reumatologia é um aspecto favorável à formação do médico e que merece ser destacado. Hayata et al. ${ }^{6}$ observou que a osteoartrite foi a doença mais encontrada no ambulatório de Reumatologia, tanto em atendimentos em consultórios particulares, como no ambiente universitário, correspondendo a cerca de $22 \%$ dos casos estudados. Embora seja uma doença considerada benigna, que não se associa a causa de morte na população idosa, ela merece atenção especial por sua elevada prevalência - $65 \%$ dos casos de incapacidade no Brasil segundo Machado et al. ${ }^{8}$ - e por repercutir diretamente sobre a qualidade de vida do paciente ${ }^{9}$. Assim reforça-se a importância da formação de um profissional apto a conduzir esta afecção tão comum e, com base nas competências adquiridas, otimizar a qualidade de vida dos pacientes.

Estudos epidemiológicos estimam a prevalência de AR em $1 \%$ da população adulta, afetando três vezes mais mulheres do que homens e com maior incidência entre os 35 e 65 $\operatorname{anos}^{10}$. No Brasil, um estudo multicêntrico verificou prevalência de AR do adulto variando de $0,2 \%$ a $1 \%{ }^{11}$. Diante desta prevalência e do seu consequente impacto social, é fundamen- 
tal que o acadêmico de Medicina tenha oportunidade de se familiarizar com esta entidade, como ocorre no ambulatório de Reumatologia do Cemec.

O LES deve ser destacado no grupo das colagenoses, devido a sua frequência, acometimento multissistêmico e gravidade que o quadro pode adquirir, envolto em importante morbimortalidade. A doença é mais frequente em mulheres (10:1), com pico de incidência em torno de 30 anos $^{12}$. As manifestações clínicas do LES são variadas, podendo envolver qualquer órgão ou sistema, isolada ou simultaneamente, em qualquer período da doença ${ }^{13}$. Isto, muitas vezes, dificulta seu diagnóstico e tratamento, baseados na supressão da atividade inflamatória ${ }^{14}$, havendo necessidade de o futuro médico generalista estar familiarizado com as principais facetas da doença, a fim de que haja melhor manejo dos casos.

É interessante notar que reumatismos de partes moles se apresentaram em menor número se comparados a desordens autoimunes, como a AR e o LES. A menor representação desse tipo de doença deve-se, provavelmente, à retenção dos casos de maior gravidade no serviço especializado, em detrimento de doenças que, embora de grande importância epidemiológica, não apresentam repercussão na sobrevida do paciente. Mesmo assim, ainda é válido destacar que o percentual de atendimentos que envolve este grupo de doenças foi significativo, correspondendo a $15,69 \%$ dos casos, enquanto em outro estudo este percentual não ultrapassou 2,8\% dos atendimentos em ambiente universitário ${ }^{6}$.

Graças ao aumento da expectativa de vida no último século, observado em países desenvolvidos e em desenvolvimento, a incidência e a prevalência da osteoporose e seu custo para a sociedade estão crescendo consideravelmente ${ }^{15}$. Reginster e Burlet e Reginster ${ }^{16}$ estimaram que cerca de 200 milhões de pessoas em todo o mundo apresentam osteoporose. A grande consequência desta doença é aumentar a fragilidade óssea e a suscetibilidade a fraturas, provocando aumento da mortalidade e do número de idosos acamados ${ }^{17}$. Considerando a relevância dos dados expostos e a necessidade de domínio deste tema pelo graduando, este estudo revelou que a osteoporose foi a quinta doença mais diagnosticada. Apesar da prevalência relativamente baixa, é importante considerar que outras especialidades também acompanham os casos de osteoporose, como a Ginecologia, Endocrinologia e Geriatria, além dos especialistas em Medicina de Família e Comunidade, havendo oportunidade de discussão aprofundada sobre esta entidade em diversos momentos do curso.

A fibromialgia (FM) é uma síndrome reumatológica crônica de etiologia desconhecida, manifestada predominantemente no gênero feminino através de dores difusas ${ }^{18}$. Um aprendizado consistente acerca da FM é de grande importância, especialmente ao se considerar que não existem exames complementares que a identifiquem. Portanto, mostra-se necessário o domínio do tema pelo médico generalista, destacando-se a importância da anamnese e do exame físico para esse diagnóstico. Acrescenta-se a isto sua notabilidade epidemiológica: após submeter 3.038 pessoas a exame reumatológico, Senna et al. ${ }^{19}$ constatou que a FM foi a segunda desordem reumatológica mais frequente, com prevalência de 2,5\%. Em outro estudo, a FM figurou como o segundo distúrbio mais prevalente nos consultórios de reumatologistas ${ }^{20}$.

A espondiloartrite (EA) geralmente tem início na segunda ou terceira década de vida, acometendo principalmente indivíduos do gênero masculino ${ }^{21,22}$. A prevalência da EA é de $0,1 \%-1,4 \%$, apresentando grande variação tanto geográfica quanto em relação aos grupos étnicos ${ }^{23}$. Foram identificados $4,06 \%$ de casos de EA neste estudo, resultado semelhante ao encontrado por Hayata et al. ${ }^{6}$ entre pacientes de consultórios particulares de reumatologistas (3,2\%).

De acordo com os dados obtidos, a lombalgia foi o diagnóstico de $0,87 \%$ da população estudada, e a gota, de 1,74\%. Portanto, ao identificar que doenças importantes - como artropatias por cristais, cervicalgia e lombalgia - são pouco frequentes entre os pacientes do ambulatório de Reumatologia, torna-se clara a necessidade de, aproveitando o contato com o professor especialista na área, garantir momentos de discussão sobre estas entidades, seja na forma de casos clínicos, seja em seminários, por exemplo. E tal discussão deve ocorrer de forma constante, não apenas a depender da ocorrência de pacientes com estas doenças.

\section{CONCLUSÕES}

Conclui-se que o perfil de doenças com o qual os graduandos do Cesupa têm tido maior contato é, em parte, compatível com o perfil de afecções musculoesqueléticas mais comum da prática clínica. Porém, preocupa a baixa frequência de doenças como gota, cervicalgia e lombalgia, devendo a instituição estar atenta a otimizar oportunidades de contato dos alunos com pacientes com tais doenças, propiciando a formação de um médico generalista capacitado a conduzir as principais afecções reumatológicas.

\section{REFERÊNCIAS}

1. Hossie GAC. Teaching rheumatology in primary care. Ann Rheum Dis [on line]. 2000. 59(7) [capturado em: 10 ago 2014]; 500-503. 2000. Disponível em: http:/ /ard.bmj.com/ content $/ 59 / 7 / 500$.full?ijkey=9f0c5d9e28c8a9702db0fdfb7 10caac032b1f0e8\&keytype2=tf_ipsecsha 
2. Weinstein SL. The bone and joint decade. J Bone Joint Surg Am [on line]. 2000. 82(1) [capturado em: 10 ago 2014]; 1-3. Disponível em: http://www.ncbi.nlm.nih.gov/pubmed/10653078

3. Mazzuca SA, Brandt KD, Katz BP, Li W, Stewart KD. Therapeutic strategies distinguish primary care physicians from rheumatologists in the management of osteoarthritis. J Rheumatol [on line]. 1993. 20 [capturado em: 10 ago 2014]; 80-86. Disponível em: http://www.ncbi.nlm.nih. gov/pubmed/8441172

4. Glazier RH, Dalby DM, Badley EM, Hawker GA, et al.Determinants of physician confidence in primary care management of musculoskeletal disorders. J Rheumatol [on line]. 1996. 23 [capturado em: 10 ago 2014]; 351-356. Disponível em: http://www.ncbi.nlm.nih.gov/pubmed/8882045

5. Abou-raya A, Abou-raya S. The inadequacies of musculoskeletal education. Clinical Rheumatology [on line]. 2010. 29(10) [capturado em: 10 ago 2014]; 1121-6. Disponível em: http:/ / www.ncbi.nlm.nih.gov/pubmed/20607334

6. Hayata ALS, Gonçalves CR, Borba EF, Levy-Neto M, Bonfá EDO. Comparação entre os acientes reumatológicos do hospital escola e de consultórios particulares e sua impotância na formação do especialista. Revista bras. Reumatol [on line]. 1999. 39 [capturado em: 10 ago 2014];259-262. Disponível em: http://bases.bireme.br/cgi-bin/wxislind. exe/iah/online/?IsisScript=iah/iah. $x i s \& s r c=$ google\&bas e=LILACS\&lang=p\&nextAction=lnk\&exprSearch=308753 \&indexSearch $=\mathrm{ID}$

7. Caldas CAM, Paz OAG, Negrao JNC, Caldato MCF. Rheumatology in a medicine course with problem-based learning. Rev. bras. educ. med. [online]. 2013.37(4) [capturado em: 2 ago. 2014]; 584-590. Disponível em: http:// www.scielo.br/scielo.php?script=sci_arttext\&pid=S0100$-55022013000400015 \& \operatorname{lng}=$ pt\&nrm $=$ iso

8. Machado GPM, Barreto SM, Passos VMA, Lima-Costa MFF. Projeto Bambuí: prevalência de sintomas articulares crônicos em idosos. Rev. Assoc. Med. Bras [on line]. 2004. 50(4) [capturado em: 10 ago 2014]; 367-372. Disponível em: http:/ /dx.doi.org/10.1590/S0104-42302004000400024.

9. Alves JC, Bassitt DP. Qualidade de vida e capacidade funcional de idosas com osteoartrite de joelho. [on line]. 2013. 11(2). [capturado em: 10 ago 2014]; 209-215.Disponível em: http:/ /dx.doi.org/10.1590/S1679-45082013000200013.

10. Louzada-junior P, Souza BDB, Toledo RA, Ciconelli RM. Análise descritiva das características demográficas e clínicas de pacientes com artrite reumatóide no estado de São Paulo, Brasil. Rev. Bras. Reumatol. [on line]. 2007.
47(2) [capturado em: 10 ago 2014] Disponível em: http:/ / dx.doi.org/10.1590/S0482-50042007000200002.

11. Marques Neto JF, Gonçalves T, Barros EFO, et al: Estudo multicêntrico da prevalência da artrite reumatóide do adulto em amostras da população brasileira. Rev Bras Reumatol [on line]. 1993. 33(5) [capturado em: 10 ago 2014]; 169-73; Disponível em: http:/ /bases.bireme.br/cgi-bin/wxislind.exe/iah/online/?IsisScript=iah/iah.xis\&n extAction $=\operatorname{lnk} \&$ base $=$ LILACS\&exprSearch $=169296 \&$ inde $\mathrm{xSearch}=\mathrm{ID} \&$ lang $=\mathrm{p}$

12. Danchenko N, Satia JA, Anthony MS. Epidemiology of systemic lupus erythematosus: a comparation of worldwide disease burden. Lupus [on line]. 2006. 15(5) [capturado em: 10 ago 2014]; 308-18. Disponvível em: http:/ / lup.sagepub.com/content/15/5/308.short

13. Freire EAM, Souto LM, Ciconelli RM. Medidas de avaliação em lúpus eritematoso sistêmico. Rev Bras Reumatol [on line]. 2011. 51(1) [capturado em: 10 ago 2014]; 70-80. Disponível em: http://www.scielo.br/pdf/rbr/v51n1/ v51n1a06

14. Griffiths B, Mosca M, Gordon C. Assessment of patients with systemic lupus erythematosus and the use of lupus disease activity indices. Best Pract Res Clin Rheumatol [on line]. 2005. 19(5) [capturado em: 10 ago 2014]; 685-708. Disponível em: http://www.bprclinrheum.com/article/ S1521-6942(05)00032-X/abstract

15. Kowalski SC, Sjenzfeld VL, Ferraz MB. Utilização de recursos e custos em osteoporose. Assoc Med Bras [on line]. 2001. 47(4). [capturado em: 10 ago 2014]; 352-7. Disponível em: www.scielo.br/pdf/ramb/v47n4/7403.pdf

16. Reginster JY, Burlet N. Osteoporosis: a still increasing prevalence. Bone. [on line]. 2006. 38(2). [capturado em: 10 ago 2014]; S4-9. Disponível em: www.ncbi.nlm.nih.gov/pubmed/16455317

17. National Institue of Health - NIH. Consensus Development Panel On osteoporosis prevention, diagnosis and therapy. Osteoporosis prevention, diagnosis and therapy J Am Med Assoc. [on line]. 2001. 285 [capturado em: 10 ago 2014]; 785-95. Disponível em: http://www.ncbi.nlm.nih. gov/pubmed/11176917

18. White KP, Speenchley M, Harth M, Ostbye T. Comparing self-reported function and work disability in 100 comunity cases of fibromyalgia syndrome versus controls in London, Ontario. Arthritis and Rheum [on line]. 1999; 42:76-83. Disponível em: www.ncbi.nlm.nih.gov/pubmed/9920017

19. Senna ER, De Barros AL, Silva EO, Costa IF, Pereira LV, Ciconelli RM, et al. Prevalence of rheumatic diseases in Brazil: a study using the COPCORD approach. J Rheumatol 
[on line]. 2004. 31 [capturado em: 10 ago 2014]; 594-7. Disponível em: www.ncbi.nlm.nih.gov/pubmed/14994410

20. Wolfe F, Cathey MA. Prevalence of primary and secondary fibrositis. J Rheumatol [on line]. 1983. 10. [capturado em: 10 ago 2014]; 965-8. Disponível em: www.ncbi.nlm.nih. gov/pubmed/6582268

21. Sampaio-Barros PD. Epidemiology of spondyloarthritis in Brazil. Am J Med Sci [on line].2011. 341(4) [capturado em: 10 ago 2014]; 287-8. Disponível em: www.ncbi.nlm.nih. gov/pubmed/21358306

22. Zink A, Braun J, Listing J, Wollenhaupt J. Disability and handicap in rheumatoid arthritis and ankylosing spondylitis - results from the German rheumatological database. German Collaborative Arthritis Centers. J Rheumatol [on line]. 2000. 27(3) [capturado em: 10 ago 2014]; 613-22. Disponível em: www.ncbi.nlm.nih.gov/pubmed/10743798

23. Khan MA, Van der Linden SM. Ankylosing spondylitis and other spondyloartropathies. Rheum Dis Clin North Am [on line]. 1990. 16(3) [capturado 10 agosto 2014];
551-79. Disponivel em: http://www.scielo.br/scielo. php?script=sci_arttext\&pid=S0482-50042012000500009

\section{CONTRIBUIÇÃO DOS AUTORES}

Todos os autores participaram da concepção do estudo, pesquisa de campo, análise dos dados, elaboração e revisão do artigo.

\section{CONFLITO DE INTERESSES}

Os autores declaram a inexistência de conflito de interesses.

\section{ENDEREÇO PARA CORRESPONDÊNCIA}

Cezar Augusto Muniz Caldas

Centro Universitário do Estado do Pará (Cesupa)

Av. Almirante Barroso, 3775

Souza - Belém

CEP 66613-710 - PA

E-mail: cezar_caldas@yahoo.com.br 